Analyse moyenne des gaz du charbon de bois

\begin{tabular}{|c|c|c|}
\hline & $\begin{array}{l}\text { Pour cent } \\
\text { des gaz }\end{array}$ & $\begin{array}{l}\text { Pour cent du charbon } \\
\text { de bois }\end{array}$ \\
\hline $\mathrm{CO}^{2}$ & 38,6 & 3,96 \\
\hline $\mathrm{CO}$ & 41,65 & 4,272 \\
\hline$C I^{4} \ldots \ldots \ldots \ldots \ldots$ & $\mathrm{xg}, \mathrm{o} 4$ & $x, 955$ \\
\hline \multirow[t]{2}{*}{$H \ldots \ldots \ldots \ldots \ldots \ldots$} & $0,7 \mathrm{I}$ & 0,073 \\
\hline & roo, 00 & Jo, 260 \\
\hline
\end{tabular}

Analyse moyenne des gaz secs en poids

\begin{tabular}{|c|c|c|c|c|c|}
\hline & & G & $\mathrm{O}$ & II & $\mathrm{N}$ \\
\hline $\mathrm{CO}^{2}$ & $33, \mathrm{I} 6$ & 9,06 & $24, x$ & $n$ & $"$ \\
\hline o. & 0,12 & $"$ & 0,12 & $n$ & $"$ \\
\hline Co & 60,45 & $25,9 \circ$ & $3 /, 5,5$ & $n$ & $"$ \\
\hline & 0,83 & $"$ & $"$ & 0,83 & $"$ \\
\hline $\mathrm{CH}^{4}$ & $\mathrm{r}, 4$ & $\mathrm{~T}, 05$ & $"$ & 0,35 & ") \\
\hline$\ldots \ldots \ldots$ & 4,04 & $"$ & $"$ & $n$ & 4,04 \\
\hline
\end{tabular}

\section{ÉCLAIRAGE ÉLECTRIQUE}

\section{GRANDEURS ET UNITÉS PHOTOMÉTRIQUES}

\begin{abstract}
Vu l'intérêt pratique qui s'attache à la fixation des unités de mesures photométriques, nos Lecteurs nous sauront gré de reproduire les propositions de la deuxième section du Comité de la Société Internationale des Electriciens, présentées par M. Bоснет, au cours de la séance da rer mai rgi2.
\end{abstract}

La deuxième section de nolre Comité m'a chargé de vous communiquer le résullat de l'examen des propositions relatives aux grandeurs et unités photométriques, qui ont élé soumises à notre Société. Cette question nous intéresse d'autant plus que la photométrie est une science d'origine française, dont les premières lois et les premières méthodes de mesures ont été exposées au XVIII ${ }^{\mathrm{e}}$ siècle par l'illustre Bouguer et par Lambert.

La détermination des grandeurs et unités photométriques a déjà été étudiée par les physiciens à différentes reprises ; elle a fait notamment l'objet d'une importante discussion au Congrès des Electriciens de Genève en 1896 . Je vous rappelle qu'un rapport très complet sur les Communications faites à ce sujet au Congrès de Genève a été publié cette même année dans le Bulletin de notre Société, page 35\%.

C'est à mon éminent collègue et ami, M. Blondel, que revient l'honncur des améliorations considérables apportées à la terminologie photométrique, par l'application à la lumière de définitions analogues à celles usitées pour le magnétisme. La considération du flux lumineux qui domine ces réformes est particulièrement fertile en conséquences utiles. Ces travaux ont conduil à l'élaboration, au Congrès de Genève de 1896 , d'un système cohérent ayant pour base la bougie décimale (qu'on supposait alors être représentée avec une approximation pratique suffisante par l'étalon Hefner), le mètre et la seconde. Depuis celte époque, différents changements sont survenus, parmi lesquels il convient de signaler en première ligne l'accord heureux qui s'est établi entre les Laboratoires nationaux des Etats-Unis, de la France et de l'Angleterre pour l'adoption d'une bougie internationale commune aux trois pays et dont la valeur est sensiblement la même que celle de la bougie décimale, ou bougie Violle, laquelle est égale au $\mathrm{r} / 20^{\circ}$ de l'étalon au platine défini par
M. Violle et a été adoptée comme unité photométrique par le Congrès international des Electriciens de Paris, en 1889 , sur sa proposition.

La comparaison de l'unité Hefner à la bougie décimale a été, d'autre part, l'objet de mesures très précises des différents laboratoires nationaux et, en particulier, a été faite en France par le Laboratoire central d'Electricité (MM. Laporte et Jouaust, sous la direction de M. Janet) et le Laboratoire national d'essai du Conservatoire des Arts-et-Métiers (M. Pérot). On a été conduit ainsi à établir que l'unité Hefner est, pratiquement, les 0,90 de la bougie internationale, de sorle que toutes les mesures faites avec la bougie Hefner peuvent être converties immédiatement en mesures internationales.

Le moment a donc paru venu aux spécialistes de différents pays de remettre au point le système international d'unités et de grandeurs photométriques, et cette tâche a été entreprise aux Etats-Unis par l'Illuminating Engineering Sociely, en rgro, sous la présidence de M. Humphreys.

Une Sous-Commission a été chargée de ces travaux. Rendant un juste hommage aux mérites de nos compatriotes,les Américains ont tenu à ce qu'ils fussent représentés dans cette Sous-Commission qui comprenait : MM. Sharp, président ; Blondel, Kenelly, Nichols et Rosa.

Les définitions arrêtées par ce Subcommittee on Nomenclature and Standard, et qui font l'objet de la proposition soumise à notre Société, sont portées au Tableau ci-annexé. Ces définitions diffèrent peu de celles qui ont été adoptées autrefois à Genève ; on a préféré comme unité de longueur le centimètre au mètre, afin d'obtenir un plus grand caractcre d'internationalité, étant donné que les pays anglo-saxons n'ont pas encore adopté le système métrique, mais seulement le système G. G. S. Le centimètre avait d'ailleurs été déjà adopté à Genève comme unité secondaire de longueur pour les mesures d'éclal intrinsèque ; il devient maintenant unité principale. On a, d'autre part, admis comme unité la bougie internationale.

Enfìn, on a rétabli la définition de la radiation spécifique que M. Blondel avait présentée autrefois à Genève et qui n'avait pas été acceptée ; l'expérience a démontré que cette radiation spécifique est fort utile.

Enfin, l'adoption du centimètre comme unité de longueur fondamentale a fait disparaître le lux, ou bougie-mètre, comme unité fondamentale d'éclairement, au profit du llmen par centimètre carré ; unité qui a l'avantage de porter en elle-même sa définition.

Ce Tableau, qui constitue la mise au point probablement définitive des unités photométriques, figurera dans le Recueil des constantes de la Société de Physique. Toutefois, dans cette publication, le flux lumineux est représenté par $\Phi$ et l'intensilé lumineuse par $J$, suivant un usage déjà très répandu, afin d'éviter des confusions avee la force et l'intensité du courant.

La définition des grandeurs et unités photométriques est basée sur les considérations suivantes.

La lumière se manifesle par l'excitation que produit la radiation qui provoque la vision. Le débit ou flux de lumière est proportionnel au flux de l'énergie rayonnante, et a un coefficient d'excitation qui dépend principalement de la distribution spectrale de cetle énergie. La présence du flux de lumière se constate par l'éclairement qu'il produit. L'éclairement est proportionnel au flux par unité d'aire de la surface éclairée ou à la densité du flux tombant sur cette surface. 
L'intensité lumineuse d'une source ponctuelle de lumière est mesurée par le flux lumineux émis dans un angle solide égal à l'unité, suivant la direction dans laquelle l'intensité est mesurée.

Deux sources ponctuelles de lumière se disent égales en inlensité quand elles produisenl un éclairement égal à des distances égales. Grâce au fait qu'on peut obtenir des sources ponctuelles étalonnées d'intensité lumineuse invariable, landis qu'il n'est pas pratique d'obtenir un flux lumineux étalonné, ou un éclairement étalonné sans avoir à sa disposition une source de lumière élalonnée, l'unité d'intensité lumineuse est devenue l'unité pholométrique fondamentale dont dérivenl les valeurs des unités de flux, d'éclairement, elc. etc. ; autrement dit, l'unité d'intensité est l'unité photométrique fondamentale, quoique le flux soit la grandeur photométrique fondamentale.

L'unité d'intensité lumineuse est la bougie. Par bougie, on entend la bougie commune à la Grande-Bretagne, à la France et à l'Amérique et à laquelle on a proposé de donner le nom de bougie internationale. Le terme puissance lumineuse peut être employé comme équivalent au terme intensité lumineuse en bougies.

L'unité de flux lumineux est le flux de lumière produit dans un angle solide égal à l'unité (un stéradiam) par une source uniforme de lumière de I bougie placée au sommet de l'angle. Cetle unité est appelée lumen. Le lumen est donc indépendanl des unités métriques. Cette particularité en a facilité l'adoption générale.

L'unité d'éclairement est l'éclairement qui est produit par un flux de I lumen tombant sur une surface plane égale ì l'unité. Cet éclairement est égal à celui qui est produit par une source de I bougie placée à l'unité de distance. L'unité d'éclairement dans le système C. G. S. est le lumen par centimètre carré. Comme unité pratique, on recommande le millilumen par centimètre carré, qui est égal à to bougiesmètres, ou à I lumen par mètre carré, ou bien encore à Io lux, lerme qu'il serait intéressant de conserver.

Par intensité spécifique d'une surface ou corps lumineux, expression qui remplacera désormais avantageusement celle beaucoup moins claire d'éclat intrinsèque, on entend, suivant les principes généraux de la nomenclature physique, une intensité lumineuse apparente par unité de surface exprimée en bougies par centimètre carré de surface apparente, c'cst-à-dire de surface projetée sur un plan perpendiculaire à la direction suivant laquelle elle est vue par l'observateur. Cela en considérant seulement une surface de dimensions petites par rapport à la distance à laquelle est faite la mesure. Il en résulte que pour les surfaces lumineuses dont l'émission suit la loi du cosinus, l'intensité spécifique est indépendante de la direction de visée.

Par radiation spécifigue d'une surface ou corps lumineux. on entend son flux lumineux total par unité de surface exprimée en lumens par centimètre carré, ou en millilumens par centimètre carré. Pour les surfaces lumineuses dont l'émission suit la loi du cosinus, la radiation spécifique est égale à l'intensité spécifique multipliée par $\pi$.

Par intensité moyenne sphérique d'une source de lumière, on entend la valeur moyenne de son intensité mesurée dans toutes les directions de l'espace ; elle est égale au quotient du flux lumineux total exprimé en lumens par $4 \pi$.

Par facteur de réduction sphérique d'une source de lumière, on entend le rapport de son intensité moyenne sphérique à son intensité moverne horizontale.

Les noms de ces unités dans le système C. G. S. et, leurs relations mathématiques entre elles sont résumées dans le Tableau qui suit :
I. Intensité de lumière ou puis-
sance de source lumineuse.
Bougie
internationale
$I=\frac{\mathrm{d} F}{\mathrm{~d} \omega} ; I_{s}=f I_{h}$
2. Flux lumineux.......... Lumen.
$F=4 \pi I_{s} ; F=I \omega$.
3. Eclairement.$\ldots \ldots \ldots \ldots \frac{\text { Lumens }}{\mathrm{cm}^{2}}{ }^{(1)}$
$E=\frac{F}{S}=\frac{I}{r^{2}}$.
4. Radiation spécifique ...... $\frac{\text { Lumens }}{\mathrm{cm}^{2}}$.
$R=\frac{F}{S}=m E$.
5. Intensité spécifique........ $\frac{\text { Bougies }}{\mathrm{cm}^{2}}$.
$e=\frac{I}{S\left({ }^{2}\right) \cos \varepsilon}$.
6. Intensité spécifique....... \{ $\begin{gathered}\text { Lumen-heure ou } \\ \text { Lumen-seconde. }\end{gathered} Q F E$.

La bougie internationale est représentée en abréviation par $\mathrm{C}$; Le lumen international est représenté en abréviation par L;

L'unité Hefner est représentée en abréviation par HK, suivant

l'usage actuel de l'Allemagne ;

$f$ est le facteur de réduction sphérique d'une source de lumière;

w est un angle solide airc d'une sphère sous-tendant un angle

égal au rapport

carré du rayon

$r$ est la distance de la sourcc à la lumière en centimètres;

$m$ est le coefficient de réflexion ou de radiation;

( $\mathrm{s}-m=$ absorption) ;

$S$ est une aire mesurée en centimètres;

$e$ est un angle d'émission; et $i$ est un angle d'incidence.

Les membres de la deuxième section ont unanimement approuvé le Tableau des grandeurs photométriques proposé sous les auspices de l'Illuminating Engineering Society. Ils ont été heureux d'y trouver une nouvelle consécration des idées des physiciens français, et en particulier de deux de nos collègues, par une association magistralement organisée, au sein de laquelle ces études photométriques ont été longuement poursuivies par des personnalités d'une haute compétence, dans un esprit scientifique des plus élevés.

\section{INSTALLATIONS HYDRAULIQUES}

\section{AMÉNAGEMENT DU HAUT-RHONE FRANÇAIS Le projet de "Bellegarde et Malpertuis"}

Nos lecteurs se souviennent des conférences, publiées ici en juillet et août de l'année dernière, que M. Lugeon, géologue, professeur à l'Université de Lausanne, et M. DE VALBREUzE, secrétaire général de la Société Internationale des Electriciens, ont faites devant la Sociétxé b'Agriculture, Sciences et Industrie, de Lyon, sur l'aménagement du Haut-Rhône français par le projet dit de "Génissiat " dont les auteurs sont MM. Harlé, Blonder, et MaHL. Or, concurremment, existe un autre projet, dit de "Bellegarde et Malpertuis ". Il a été aussi exposé à Lyon, en des conférences retentissantes, devant la même Société, par MM. Mauvernax, Coutagne et L.-A. Martel. Notre impartialité nous impose d'en indiquer également les grandes lignes.

\section{Rappel des caractéristiques du projet de Génissiat}

Nous empruntons partiellement ce résumé au tout récent rapport présenté sur le projet de Génissiat à l'Association

(1) $\mathrm{Ou} \frac{\text { Millilumens }}{\mathrm{cm}^{2}}$.

(2) $S$ se rapporte à la surface vue du point auquel l'intensité I est rapportée. 